\title{
SUPER-RESOLUTION RECONSTRUCTION OF WHOLE-BODY MRI MOUSE DATA: AN INTERACTIVE APPROACH
}

\author{
A. Khmelinskii ${ }^{1, *}$, E. Plenge ${ }^{2, *}$, P. Kok ${ }^{1,3, *}$, O. Dzyubachyk ${ }^{1, *}$, D. H. J. Poot ${ }^{2}$, E. Suidgeest ${ }^{5}$, C. P. Botha ${ }^{3}$, \\ W. J. Niessen ${ }^{2,4}$, L. van der Weerd ${ }^{5,6}$, E. Meijering ${ }^{2}$ and B. P. F. Lelieveld ${ }^{1,3}$ \\ ${ }^{1}$ Division of Image Processing, Dept. of Radiology, LUMC, Leiden, The Netherlands \\ ${ }^{2}$ BIGR, Depts. of Radiology and Medical Informatics, Erasmus MC, Rotterdam, The Netherlands \\ ${ }^{3}$ Dept. of Intelligent Systems, TU Delft, The Netherlands \\ ${ }^{4}$ Quantitative Imaging Group, Faculty of Applied Sciences, TU Delft, The Netherlands \\ ${ }^{5}$ Dept. of Radiology, MILL, Section Nuclear Medicine, LUMC, Leiden, The Netherlands \\ ${ }^{6}$ Depts. of Anatomy \& Embryology, LUMC, Leiden, The Netherlands
}

\begin{abstract}
Super-resolution reconstruction (SRR) is a post-acquisition method for producing a high-resolution (HR) image from a set of lowresolution (LR) images. However, for large volumes of data, this technique is computationally very demanding and time consuming. In this study we focus on the specific case of whole-body mouse data and present a novel, integrated, end-to-end approach to overcome this problem. We combine articulated atlas-based segmentation and planar reformation techniques with state-of-the-art in SRR to produce high resolution, interactively selected, localized isotropic volumes-of-interest in whole-body mouse MRI. With this method we overcome time and memory related limitations when applying the SRR algorithm to the entire dataset, enabling interactive visualization and exploration of anatomical structures of interest in whole-body MRI mouse data on a normal desktop PC.
\end{abstract}

Index Terms - Whole-Body, Articulated, Atlas-Based, Registration, Skeleton Segmentation, Image Processing, Molecular Imaging, Planar Reformation, Interactive, MOBY, MRI, SuperResolution Reconstruction

\section{INTRODUCTION}

SRR is the process of producing a high-resolution (HR) image from a sequence of low-resolution (LR) images, where each LR image transforms and samples the HR scene in a distinct fashion. The idea was first introduced in the 1980s [1] and has since grown into a research field of its own. The first example of SRR applied to MRI was described in a 2001 patent [2]. SRR in MRI is a developing field, and encouraging results have been published showing its potential in resolution enhancement $[3,4]$. For an overview of SRR research in the biomedical field, see [5].

One drawback of the SRR technique is the demanding computation power and time that is necessary to reconstruct large volumes (whole-body mice datasets in this case). To overcome this impracticality, in this study we take advantage of recent progress in the areas of articulated atlas-based segmentation of whole-body small animal data [6-8] and MRI super-resolution reconstruction (SRR) $[9,10]$.

We present a new integrated approach that enables global exploration of whole-body mouse MRI data, with local, interactive

* A. K., E.P., P. K. and O. D. contributed equally to this work
SRR enhancement of user selected volumes-of-interest (VOIs). The idea is similar to that of well-known web-based geographical maps, where it is possible from a global overview image to zoom in on a detail of interest. Such a functionality is relevant in a biomedical setting when working with high-resolution volumetric data. In the approach presented in this paper, data is loaded on a "just-intime" basis: from a global low-resolution image the user is allowed to zoom in on a sub-volume of interest. The data needed to reconstruct the sub-volume at the requested level of resolution is collected from a database of low-resolution images and the sub-volume is reconstructed on the fly using SRR.

The sub-volumes of interest are obtained by segmenting one of the whole-body LR datasets using the semi-automatic whole-body articulated atlas-based method, first presented in [11] and applying the articulated planar reformation algorithm [8] which maps the data to a standardized atlas space.

The contributions of this paper are twofold:

- We present a new method for producing highly resolved, user selected, localized isotropic VOIs in whole-body mouse MRI. This enables interactive visualization and exploration of anatomical structures in whole-body MRI mouse data on a normal desktop PC.

- We realise this by combining recent progress in articulated atlas-based segmentation and planar reformation with state of the art in SRR.

In the following we describe the components of our approach and show that it enables interactive reconstruction of high-resolution volumes for interactive exploration of whole-body MRI mouse data.

\section{METHODS AND MATERIALS}

\subsection{MRI mouse data}

A whole-body scan of a post-mortem $C 57 B L / 6,6$ month old, male mouse was acquired on a 7T Bruker Pharmascan ${ }^{\mathrm{TM}}$ system using a recovery FSE (frFSE) sequence. TR was $6648 \mathrm{~ms}$, TE was $33 \mathrm{~ms}$, with Navg $=1$ and NSP $=1$. The 2D slice stack consisted of 64 slices $(0.5 \mathrm{~mm}$ thick), with a FOV of $50 \times 32 \mathrm{~mm}$, and a resulting resolution of $0.125 \times 0.125 \times 0.5 \mathrm{~mm}$. The scan time per stack was $213 \mathrm{~s}$. The slice stack was rotated 24 times in uniform increments of 180/24 degrees. Due to the scanner's limited FOV, the mouse was scanned in three sections (head, chest, lower abdomen). 


\subsection{Stitching}

In order to reconstruct the complete mouse volume, one needs to know the relative positioning of each of the acquired subvolumes in the scanner. That requires registering two image stacks to each other, which, in case when only the translation is unknown, is usually done by using correlation-based methods. For the given type of data, the image gradient is the most complete and reliable source of information since MR is known to provide good contrast between different soft tissues, while direct usage of the intensity information might be hampered by possible field inhomogeneity and variations between different image stacks. In this work, we used a 3D extension of the gradient-correlation-based scheme recently developed by Tzimiropoulos et al. [12] for recovering the translations between each two consecutive image stacks. After the relative positions of image stacks are known, the final volume is obtained by using the multiresolution method of Burt and Adelson [13] in the overlap areas, see Figure 1.

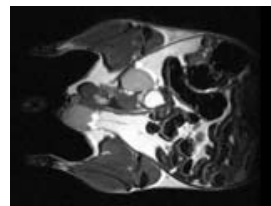

(a)

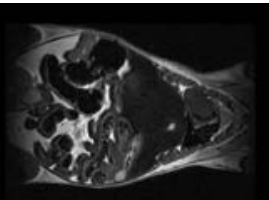

(b)

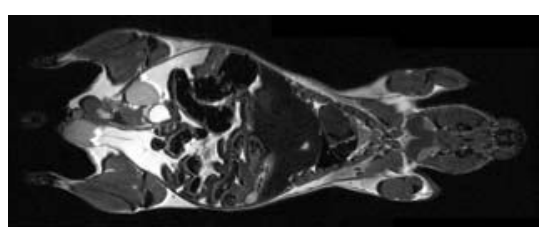

(d)

Fig. 1. Registration performance of the full volume reconstruction algorithm. One coronal slice from each of the three acquired image stacks (top 3 images) and from the final reconstructed volume (bottom) are shown. Image contrast is enhanced for visualization purposes.

\subsection{MOBY atlas}

The MOBY mouse atlas was used as the anatomical reference. Segars et al. [14] used a $C 57 B L / 6,15$ week old male mouse to generate a realistic $4 \mathrm{D}$ digital mouse phantom based on highresolution 3D MRI data. For the bone segmentation described here, we used an articulated version of the MOBY skeleton $[6,7]^{1}$, where all major bones or bone compounds like the paws were separately labeled and joint locations and types were defined. See Figure 2.(a).

\subsection{Articulated atlas-based bone segmentation}

To segment the mouse, the semi-automated bone approximation method first presented in [11] for whole-body $\mu$ MRI mouse data was applied on one of the 24 datasets (Figure 2.(b)). This approach, where the user identifies 16 landmarks, considerably reduces the required user effort compared to a manual segmentation.

Using the Amira ${ }^{\mathrm{TM}}$ software and guided by an anatomical text book [15], the following 16 joint and bone landmarks were manually extracted in one of the mouse datasets: 2 knee joints, 2 hip joints, posterior and anterior extremities of the sternum, right and left anterior pelvis extremities, 2 elbow joints, 2 shoulder joints, 2 ankle

${ }^{1}$ Publicly available for download at the Articulated Atlases Download section of the www.lkeb.nl webpage joints and 2 wrist joints (Figure 2.(c)). After the landmark selection in an arbitrary order the joints are automatically identified. Using the atlas (joint locations, anatomically realistic bone dimensions, anatomically realistic degrees of freedom for each joint) and a hierarchical anatomical model of the skeleton all joints can be labeled and the correspondent bones fitted to the data (see [11] for more details), (Figure 2.(d)).

\subsection{Articulated planar reformation}

Articulated planar reformation (APR), is a method first developed for follow-up data in [8]. This method uses the articulated registration approach described above and in [6] to reformat the data into segments corresponding to a mouse atlas and thus maps the data to a standardized atlas space. After applying APR to one of the lowresolution MRI datasets, the user can interactively select any subvolume of interest for a subsequent interactive SRR of that volume, see Figure 2.(e, f).

\subsection{Super-resolution reconstruction}

After interactive selection of a sub-volume of interest in the lowresolution MR image, spatially corresponding volumes are extracted from all low-resolution datasets. These volumes differ, according to the acquisition scheme described above, in angular shifts in the sampling grid orientation. Our super-resolution technique then is applied to reconstruct the volume on a high-resolution grid.

The MRI acquisition process can be modeled by a linear system A encompassing a geometric transformation, blur and sampling operators, and a Gaussian noise model [16]. The $k^{\text {th }}$ acquisition in a series of LR images can thus be described by $\mathbf{y}_{k}=\mathbf{A}_{k} \mathbf{x}+\mathbf{n}_{k}$, where $\mathbf{x}$ is the HR scene to be reconstructed.

$\mathrm{SRR}$ is an inverse problem and involves recovering $\mathbf{x}$ given the $\mathbf{y}_{k}$ and $\mathbf{A}_{k}$. Using additional prior knowledge about the solution, e.g. that it is smooth, the problem can be formulated as a regularized least squares problem:

$$
\mathbf{x}=\underset{\mathbf{x}}{\operatorname{argmin}} \sum_{k=1}^{N}\left\|\mathbf{y}_{k}-\mathbf{A}_{k} \mathbf{x}\right\|^{2}+\lambda \mathbf{C},
$$

where $\mathbf{C}$ is a regularization term formalizing the prior, and $\lambda$ is a scalar weight.

A direct solution of Eq. (1) is generally infeasible. Instead, iterative methods are applied to approximate the solution. For the purpose of both effective and efficient reconstruction, the method developed in [10] was chosen for our pipeline. Based on the assumption that the HR image is mainly smooth, this method employs Tikhonov regularization using the $L_{2}$-norm of the second order derivative of the reconstruction as the regularization term:

$$
\mathbf{C}=\left\|\nabla^{2} \mathbf{x}\right\|^{2}=\left(\frac{\partial^{2} \mathbf{x}}{\partial r_{1}^{2}}\right)^{2}+\left(\frac{\partial^{2} \mathbf{x}}{\partial r_{2}^{2}}\right)^{2}+\left(\frac{\partial^{2} \mathbf{x}}{\partial r_{3}^{2}}\right)^{2},
$$

where $r_{i}$ is the spatial dimension over which the partial derivative is taken. The regularized LS problem of Eq. (1) was solved by the conjugate gradient method and the transforms in $\mathbf{A}$ and $\mathbf{A}^{T}$ were implemented using a set of shear transforms that minimizes aliasing and spectral distortions [10].

\section{EXPERIMENTAL RESULTS}

The proposed SRR approach was tested for each segmented bone (femur, tibia-fibula, pelvis, sternum, humerus, ulna-radius), using an increasing number of LR images (2, 4, 8 and 12). The SRR times for each bone experiment were compared with the SRR times for the 


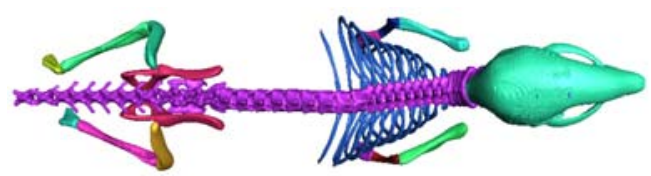

(a)

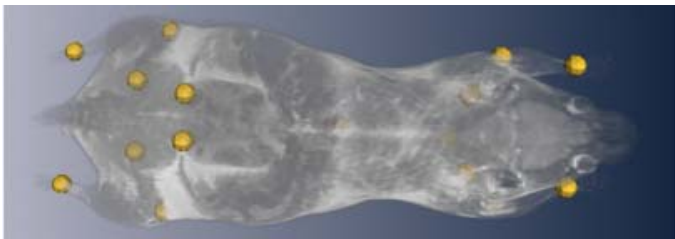

(c)

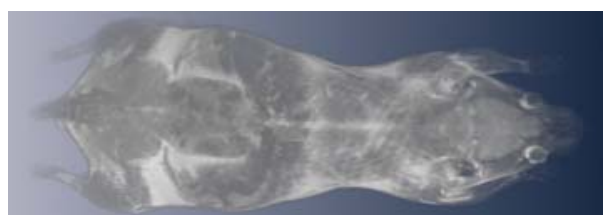

(b)

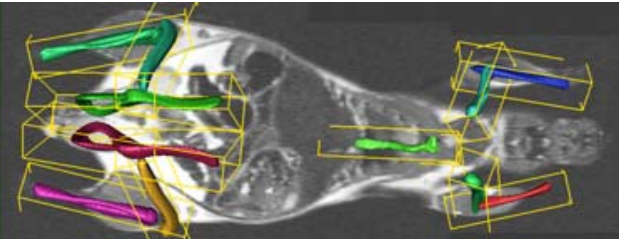

(d)
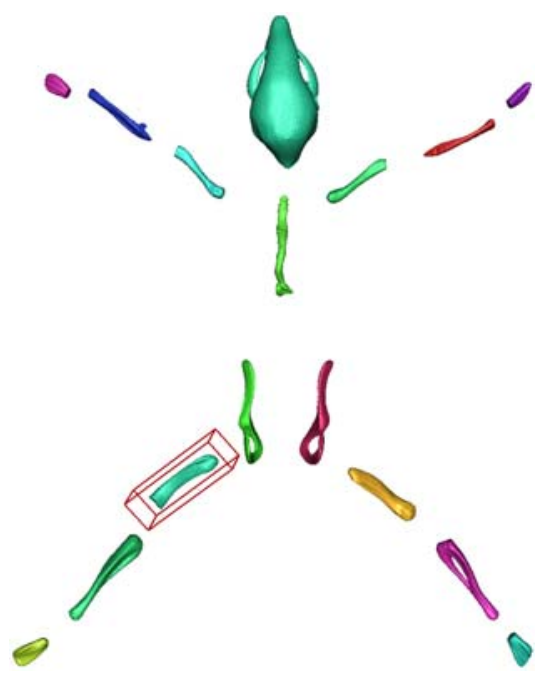

(e)
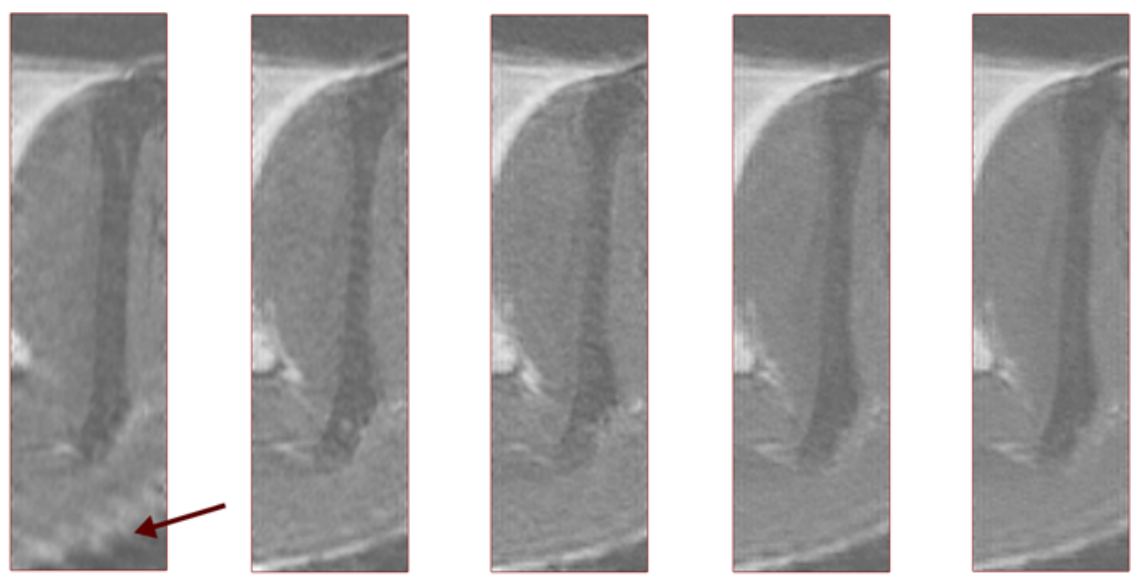

(f)

Fig. 2. Overview of the proposed approach: (a) - MOBY mouse atlas: articulated skeleton [7,14]. (b) - Original, low-resolution, stitched MRI data. (c) - Manual landmarks selection: 2 knee joints, 2 hip joints, posterior and anterior extremities of the sternum, 2 elbow joints, 2 shoulder joints, 2 ankle joints, 2 wrist joints, right and left anterior pelvis extremities. (d) - Registered atlas bones to the data. (d, e) - APR of the LR, MRI mouse data. The user can now interactively select any LR bone of interest (left femur) from the standardized MOBY atlas space. (f) The SRR algorithm applied to the selected left femur. From left to right: the selected LR image and the HR image reconstructed using $2,4,8$ and 12 LR images respectively. The improvement in image quality is especially noticeable when using a high zooming factor. The red arrow indicates the distortion caused by the low sampling in the $z$ direction during the acquisition. Applying the SRR, results in HR volumes with isotropic sampling and this issue is resolved. Note: the $z$ axis of the volume shown is not aligned with the $z$ direction during acquisition but with the principal axes of the bone. The data has been resampled in order to align the bone to the reconstructed volume. 
same experiments performed for the whole-body dataset. The times to perform each SRR experiment are shown in Table 1. All the experiments were implemented in MATLAB $R 2009 b^{\mathrm{TM}}$ and performed on a $2.80 G H z$ Intel Xeon ${ }^{\mathrm{TM}}$ with $12 G B$ of RAM, Windows ${ }^{\mathrm{TM}}$ PC.

\begin{tabular}{|c|c|c|c|c|}
\cline { 2 - 5 } \multicolumn{1}{c|}{} & $2 \mathrm{LR}$ & $4 \mathrm{LR}$ & $8 \mathrm{LR}$ & $12 \mathrm{LR}$ \\
\hline Femur & 52 & 75 & 140 & 222 \\
\hline Tibia-Fibula & 59 & 94 & 184 & 286 \\
\hline Pelvis & 84 & 119 & 249 & 372 \\
\hline Sternum & 64 & 77 & 150 & 216 \\
\hline Humerus & 41 & 58 & 117 & 170 \\
\hline Ulna-Radius & 55 & 84 & 159 & 249 \\
\hline Whole-Body & 3934 & 7596 & 14603 & NA \\
\hline
\end{tabular}

Table 1. SRR times in seconds, for each reconstructed bone and the whole-body, using 2, 4, 8 and 12 LR images. For the whole-body experiments, the SRR times using $12 \mathrm{LR}$ images were not possible to compute due to insufficient memory.

Figure 2.(f) shows the results of the experiments on the left $f e$ mur of the post-mortem mouse. The sample axial slices demonstrate that the quality of the reconstructions clearly improves when an increasing number of LR images is used. It can be observed that, in agreement with the results of the quantitative experiments [17], the quality improvement is substantial going from 2 to 12 LR images (the improvement in image quality is especially noticeable when using a high zooming factor). Though not presented in this paper, it was previously shown in [17] that a smaller effect is seen when going from 12 to 24 LR images. Again, the highest resolution is achieved by direct acquisition with the slice of interest in-plane.

SRR times scale approximately linearly with the size of the LR dataset. Since one LR image of the entire mouse contains $322 \times 803 \times 80=20,685,280$ voxels, and a typical VOI contains $100 \times 100 \times 25=250,000$ voxels, we obtain an increase of approximately a factor 100 . Looking at Table 1 and Figure 3, one also sees that the SRR times scale approximately linearly with the number of LR images used. While the entire mouse requires hours to reconstruct, the VOI can be reconstructed within minutes.

\section{DISCUSSION AND CONCLUSION}

In this paper, an integrated approach to interactively SRR reconstruct user selected VOIs was presented. It allows to overcome the time and memory limitations of the SRR technique. However, as shown above, the mean time for the best quality SRR result, i.e., using 12 LR images, is still in the order of minutes $(252.5 \mathrm{~s})$. This result is still far from the real-time target for this approach. Since the re-

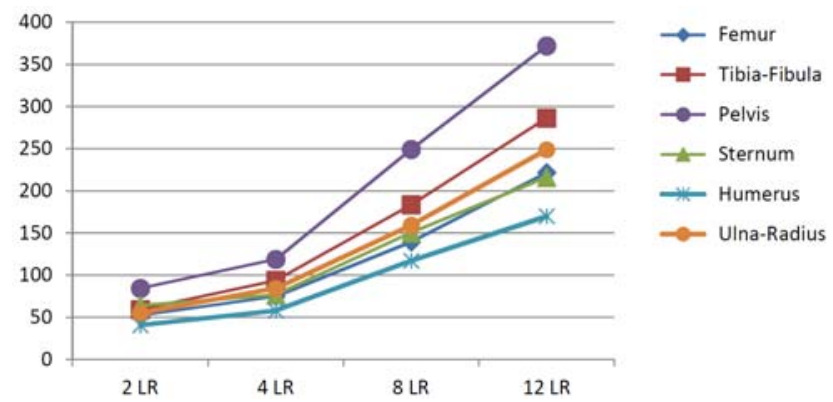

Fig. 3. SRR times in seconds, for each reconstructed bone, using 2, 4, 8 and 12 LR images. sults presented here were acquired on a MATLAB ${ }^{\mathrm{TM}}$ implemented prototype, the computation times will considerably decrease by reimplementing the algorithm in a $\mathrm{C} / \mathrm{C}++$ and GPU programming environment combination.

\section{ACKNOWLEDGMENTS}

The authors gratefully acknowledge Medical Delta for the financial support.

\section{REFERENCES}

[1] R. Y. Tsai and T. S. Huang, "Multi-frame image restoration and registration," in Advances in Computer Vision and Image Processing, vol. 1, pp. 317-339. JAI Press Inc., 1984.

[2] D. Fiat, "Method of enhancing an MRI signal," 2001, United States Patent 6294914.

[3] H. Greenspan et al., "MRI inter-slice reconstruction using super-resolution," Magn Reson Imaging, vol. 20(5), pp. 437446, 2002.

[4] A. Gholipour et al., "Robust super-resolution volume reconstruction from slice acquisitions: Application to fetal brain MRI," IEEE T Med Imaging, vol. 29(10), pp. 1739-1758, 2010.

[5] H. Greenspan, "Super-resolution in medical imaging," Comput $J$, vol. 52(1), pp. 43-63, 2009.

[6] M. Baiker et al., "Atlas-based whole-body segmentation of mice from low-contrast micro-CT data," Med Image Anal, vol. 14(6), pp. 723-737, 2010.

[7] A. Khmelinskii and M. Baiker et al., "Articulated whole-body atlases for small animal image analysis: Construction and applications," Mol Imaging Biol, vol. 13(5), pp. 898-910, 2011.

[8] P. Kok et al., "Articulated planar reformation for change visualization in small animal imaging," IEEE T Vis Comput Gr, vol. 16(6), pp. 1396-1404, 2010.

[9] R. Z. Shilling et al., "A super-resolution framework for 3-D high-resolution and high-contrast imaging using 2-D multislice MRI," IEEE T Med Imaging, vol. 28(5), pp. 633-644, 2009.

[10] D. H. J. Poot et al., "General and efficient super-resolution method for multi-slice MRI," in Proc. 13th MICCAI: Part I, Berlin, Heidelberg, 2010, pp. 615-622, Springer-Verlag.

[11] A. Khmelinskii et al., "Atlas-based organ \& bone approximation for ex-vivo $\mu$ MRI mouse data: a pilot study," in Proc. IEEE ISBI, 2010, pp. 1197-1200.

[12] G. Tzimiropoulos et al., "Robust FFT-based scale-invariant image registration with image gradients," IEEE T Pattern Anal, vol. 32, pp. 1899-1906, 2010.

[13] P. J. Burt and E. H. Adelson, "A multiresolution spline with application to image mosaics," ACM Transactions on Graphics, vol. 2, pp. 217-236, 1983.

[14] W. P. Segars et al., "Development of a 4D digital mouse phantom for molecular imaging research," Mol Imaging Biol, vol. 6(3), pp. 149-159, 2004.

[15] I. Bab et al., Micro-Tomographic Atlas of the Mouse Skeleton, Springer, 2007.

[16] H. Gudbjartsson and S. Patz, "The Rician distribution of noisy MRI data," Magn Reson Med, vol. 34(6), pp. 910-914, 1995.

[17] E. Plenge et al., "Super-resolution methods in MRI: Can they improve the trade-off between resolution, signal-to-noise ratio, and acquisition time?," Magn Reson Med, in press, 2012. 